\title{
Search for dark photons as candidates for Dark Matter with FUNK
}

\author{
A. Andrianavalomahefa ${ }^{a}$, K. Daumiller ${ }^{a}$, B. Döbrich ${ }^{b}$, R. Engel ${ }^{* a}$, J. Jaeckel ${ }^{c}$, \\ M. Kowalski ${ }^{d e}$, A. Lindner ${ }^{d}$, H.-J. Mathes ${ }^{a}$, J. Redondo ${ }^{f}$ M. Roth ${ }^{a}$, C.M. Schäfer ${ }^{a}$, \\ T. Schwetz ${ }^{a}$, R. Ulrich ${ }^{a}$, D. Veberič ${ }^{a}$
} [The FUNK Experiment]

${ }^{a}$ Institute for Nuclear Physics, Karlsruhe Institute of Technology (KIT), Karlsruhe, Germany

${ }^{b}$ Physics Department, CERN, Geneva, Switzerland

${ }^{c}$ Institute for Theoretical Physics, Heidelberg University, Germany

${ }^{d}$ Deutsches Elektronen Synchrotron (DESY), Zeuthen, Germany

${ }^{e}$ Department of Physics, Humboldt University, Berlin, Germany

${ }^{f}$ Department of Theoretical Physics, University of Zaragoza, Spain

*E-mail: ralph.engel@kit.edu

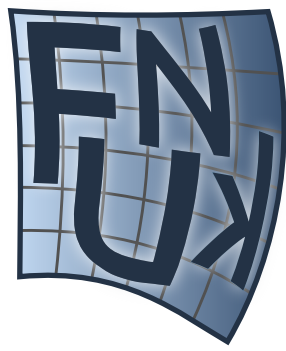

\begin{abstract}
An additional U(1) symmetry predicted in theories beyond the Standard Model of particle physics can give rise to hidden (dark) photons. Depending on the mass and density of these hidden photons, they could account for a large fraction of the Dark Matter observed in the Universe. When passing through an interface of materials with different dielectric properties, hidden photons are expected to produce a tiny flux of photons. The wavelength of these photons is directly related to the mass of the hidden photons. In this contribution we report on measurements covering the visible and near-UV spectrum, corresponding to a dark photon mass in the eV range. The data were taken with the FUNK experiment using a spherical mirror of $\sim 14 \mathrm{~m}^{2}$ total area built up of 36 aluminum segments.
\end{abstract}

36th International Cosmic Ray Conference - ICRC2019

July 24th-August 1st, 2019

Madison, WI, U.S.A.

* Speaker. 


\section{Introduction}

Our current understanding of cosmology requires that a sizable fraction of the matter content of the observable universe today is cold and hidden, thus termed cold Dark Matter (CDM). For decades, most of the experimental efforts favored the search for a whole category of new heavy particles, known as weakly interacting massive particles (WIMPs) but with no results. Only recently, more attention has been devoted to search for the lower-mass alternatives, known as weakly interacting slim particles (WISPs) which, besides other fundamental theoretical motivations, also provide natural CDM candidates [1]. Axions and massive hidden photons (HP) figure amongst the popular WISPs. Our experiment is searching for HP particles as Dark Matter.

HP can be non-thermally produced in the early universe and survive as a CDM condensate until present [2]. At low energies, HP exclusively couple with regular photons through a weak kinetic mixing with an effective Lagrangian

$$
-\mathscr{L}_{\text {eff }}=\frac{1}{4}\left(F_{\mu v} F^{\mu v}+X_{\mu v} X^{\mu v}\right)-J_{\mu} A^{\mu}-\frac{m_{\tilde{\gamma}}}{2} X_{\mu} X^{\mu}+\frac{\chi}{2} F_{\mu v} X^{\mu v},
$$

where $F_{\mu v}=\partial_{\mu} A_{v}-\partial_{v} A_{\mu}$ and $X_{\mu \nu}=\partial_{\mu} X_{v}-\partial_{v} X_{\mu}$ are the visible and hidden field strength tensors, respectively. $J_{\mu}$ is the ordinary charge current. $m_{\tilde{\gamma}}$ denotes the HP mass and $\chi$ is the mixing parameter. This model produces a large phase space compatible with CDM signatures. In recent years considerable progress has been made to constrain the viable parameter space from both astrophysical and cosmological observations as well as from laboratory experiments which are rather dedicated for axion searches [3].

The FUnK (Finding U(1)s of Novel Kind) experiment, based on the idea of a dish antenna proposed in [4], was built to search for HP signature in the DM halo. The detection principle relies on the HP-to-photon coupling which allows the DM field to carry a very tiny but ordinary photonfield. Such a mixing results in a certain probability to emit a measurable electromagnetic field upon transition between two dielectric media with different refractive indices (e.g. air-conductor).

This emitted wave is our DM signal. It oscillates at a frequency $\omega \approx m_{\tilde{\gamma}}$ and propagates in a direction almost perpendicular to the interface with an angular offset suppressed by the DM momentum [5]. The average power $P$ collected from a surface with an effective area $A_{\text {eff }}$ reads

$$
P \sim 10^{-19} \mathrm{~W}\left(\frac{\chi}{10^{-12}}\right)^{2}\left(\frac{\rho_{\mathrm{CDM}}}{0.3 \mathrm{GeV} / \mathrm{cm}^{3}}\right)\left(\frac{A_{\mathrm{eff}}}{\mathrm{m}^{2}}\right),
$$

where $\rho_{\mathrm{CDM}}$ denotes the local DM energy density. A quick inspection of Eq. (1.2) reveals that an enhancement of the signal power can be achieved with a larger area. The directionality of the signal, on the other hand, suggests the use of a spherical shape, hence a dish antenna. This configuration benefits both from a geometrical amplification of the signal and a reduction of the background ${ }^{1}$. A similar experiment, although at a smaller scale, has been previously reported in [6].

\section{Experimental setup}

The design, assembling and testing phases of FUNK are thoroughly discussed in [7, 8]. Below, we summarize the main aspects of the experiment.

\footnotetext{
${ }^{1}$ Signals are focused at the sphere radius-point, while background photons originating from far-away are gathered at the focal point.
} 

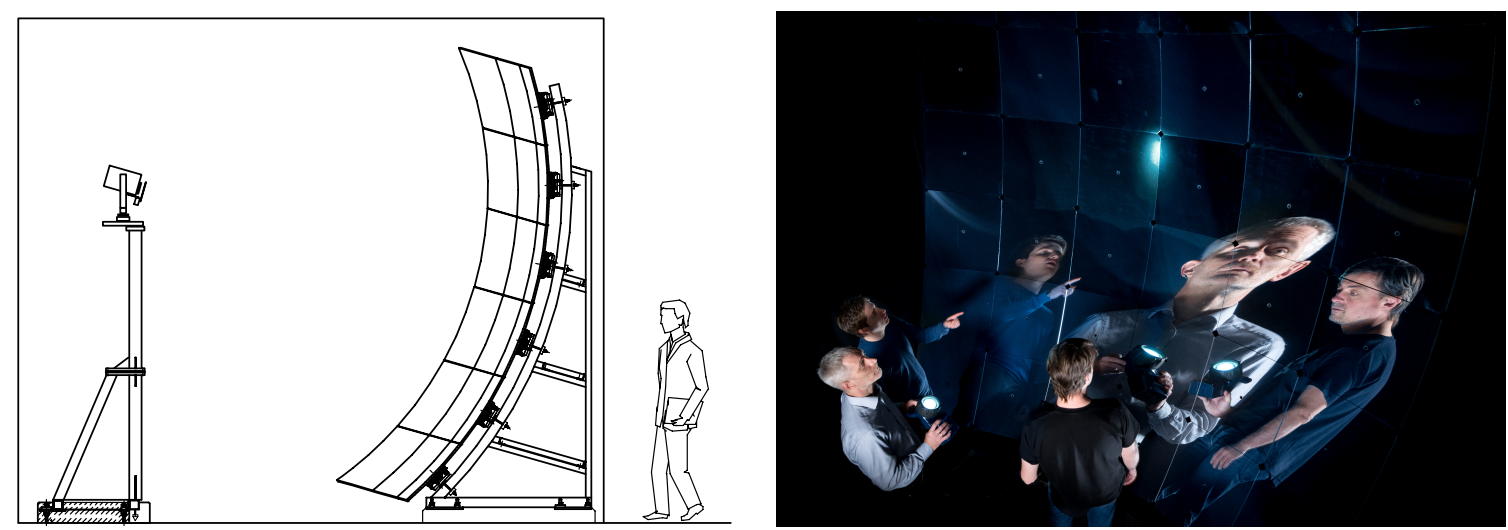

Figure 1: Left: Schematics of the FUNK setup with the detector mounted on the pillar located at the center of curvature of the sphere. Right: Picture of the mirror after assembly of all the segments.

Mirror. As a dish antenna, we reuse a prototype metallic mirror originally designed for the fluorescence telescopes of the Pierre Auger Observatory. This mirror has a spherical geometry with a radius $r \approx 3.4 \mathrm{~m}$ and is built-up out of $6 \times 6$ aluminum segments coated with a thin layer of AlMgSi, resulting in a overall reflectivity of $\sim 80 \%$ within the optical and near-UV range of frequencies. The total reflecting area is $A_{\text {mirror }} \approx 14.56 \mathrm{~m}^{2}$. The mirror has a point-spread function with a spot-size of $2 \mathrm{~mm}$ radius for $90 \%$ of the expected light at the center of curvature.

Facility. We assembled the setup inside a windowless air-conditioned experimental hall surrounded by $2 \mathrm{~m}$ thick concrete walls. The main experimental zone takes up a volume of $4.35 \times$ $4.98 \times 4.30 \mathrm{~m}^{3}$ and has been enclosed with a double-layer light-shielding consisting of a blackpolyethylene sheet over a thick-cotton curtain. Our installation also allows us to continuously monitor the environmental conditions inside the hall (ambient temperature, air pressure, muon rate) during the entire measurement campaign.

Detector. We employ a low-noise photomultiplier tube (PMT, ET-9107QB) which has an extended sensitivity range from 160 to $630 \mathrm{~nm}$ with a peak quantum efficiency of $\sim 25 \%$ at $350 \mathrm{~nm}$. The active area of the Bialkali photocathode has a diameter of $25 \mathrm{~mm}$ which is large enough to compensate for the optical aberration and capture all possible HP signals emitted from the surface of the mirror, even in the presence of seasonal movements of the signal spot [5].

Data acquisition (DAQ). The PMT output voltage is digitized by a Picoscope (6404) with a $0.8 \mathrm{~ns}$ sampling period and 8 bit analog-to-digital-converter (ADC) resolution. The acquisition is performed in self-triggered mode with a trigger-threshold of $8 \mathrm{ADC}(\sim 60 \mathrm{mV})$ below the baseline. For each captured event, a trace of 2000 bins $(0.8 \mu$ s before and after trigger $)$ is stored in a computer, together with the instantaneous environmental data. The dataset is then further analyzed off-line.

Measurement scheme. The PMT is mounted on a motorized linear stage, which can drive the camera, laterally, in or out of the position where we expect the signal. Additionally, we installed an automated optical shutter with Teflon-coated blades in front of the PMT window to control its field of view. Hence the data-taking cycles involve four measurement configurations (see Fig. 2-left), with a measurement time limited to $60 \mathrm{~s}$ (monitored to $1 \mu \mathrm{s}$ accuracy) for each of these modalities. 

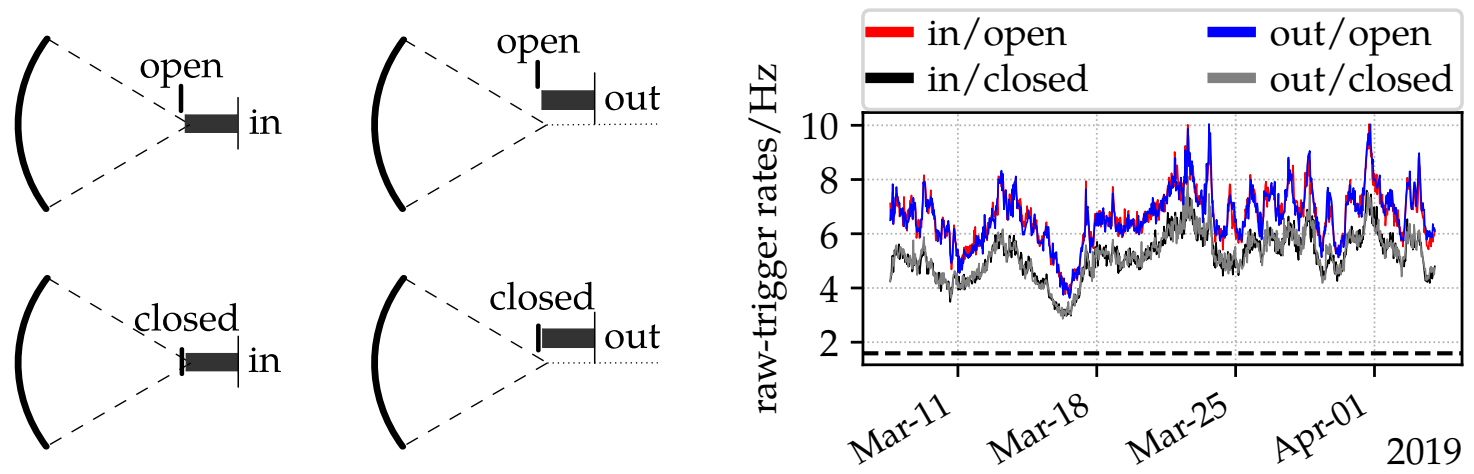

Figure 2: Left: Schematics of the four measurement configurations. Right: Raw-trigger events. Data is averaged for every 10 cycles $(\sim 44 \mathrm{~min})$. The dashed line indicates the trigger-rate level corresponding to the internal dark counts (see text for details).

\section{Analysis}

Some preliminary results were previously reported in [9]. For the analysis discussed here, we use our run v35, which was taken from March 07 to April 04, 2019 (27.5 days). Due to the measurement scheme, this corresponds to an effective live time of $145.3 \times 4 \mathrm{~h}$. In Fig. 2-right, we plot the raw-trigger rates recorded for the four measurement configurations. There are two remarks worth to mention. First, we measured the internal background ${ }^{2}$ of the PMT during an independent run, prior to v35. This was achieved by fixing a thick metal lid onto the PMT window in such a way that it remains fully light-proof during the monitoring. The result shows a very stable and low dark trigger-rate with a mean of $1.6 \mathrm{~Hz}$ and a standard deviation of $0.2 \mathrm{~Hz}$. This level is indicated by the dashed-line drawn in Fig. 2-right. Second, it is interesting to notice that the mean trigger rate measured with the shutter closed ${ }^{3}$ is, however, not only higher than the quoted internal-background, but it also follows closely the measurement taken with the shutter open. We will discuss a possible origin of this phenomenon later.

Event selection. Given the low signal power expected, our goal is to achieve a precise singlephoton electron (SPE) counting. We first performed a series of SPE calibrations by using a faint blue LED flasher whose intensity is adjustable. For each setting, the LED flashes 5000 times and each flash triggers a capture of a $1.6 \mu$ s trace onto which we run our pulse-analyzer software. The result from this flasher run is plotted in Fig. 3-left, where we can observe a saturation in the anode charge as the LED intensity gradually increases and more photons are captured. For the SPE study, the optimal settings were chosen so that (i) the PMT sees photons $20 \%$ of the time - (ii) $95 \%$ of the captured trace contain only a single pulse - (iii) the photon arrival time is estimated around $290 \mathrm{~ns}$ after the flash trigger. The corresponding charge distribution is drawn with the red line in Fig. 3left. The SPE phase space is then refined by running the calibration with this setting, which yields the region of interest (ROI) seen in Fig. 3-right. Events are selected according to the rise time, width, and Shanon entropy ${ }^{4}$ of each pulse. The last two criteria are chosen to $(i)$ avoid direct cuts

\footnotetext{
${ }^{2}$ We make a distinction between dark-noise originating from the internal parts of the PMT or from external radiation.

${ }^{3}$ The efficiency of the shutter has been tested and proven to be close to $100 \%$.

${ }^{4}$ This is done by treating each pulse as a probability mass function.
} 

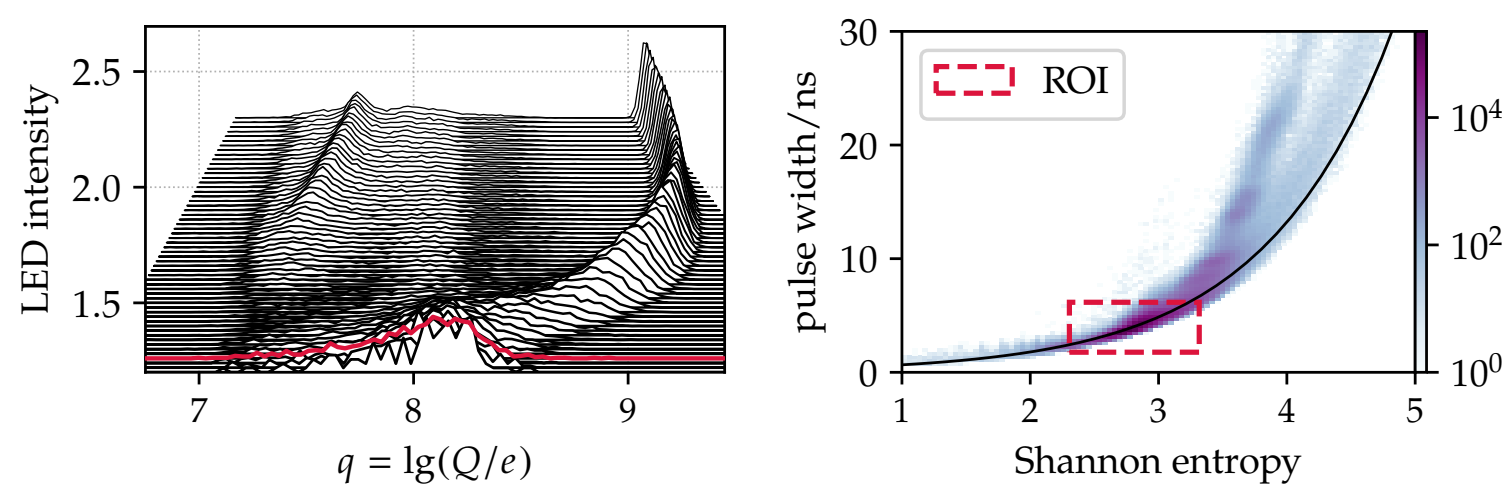

Figure 3: Left: Charge collected at the anode of the PMT as function of the intensity of the LED flasher (in arbitrary unit). The red line indicates the settings used for SPE study (see text for details). Right: 2D histogram of the pulse width and the corresponding Shanon entropy. Data were taken from the out/open configuration. The region of interest (ROI) containing SPE events is delimited by the dashed rectangle. The solid line is only indicative of the expectation for pulses whose shape is Gaussian-like.

on the pulse height or charge which is rather sensitive to the PMT gain whose standard deviation is known to be comparable to its mean - (ii) discriminate longer pulses which are most likely associated to Cherenkov-photon bursts. The cuts on rise time serve to discard spurious triggers possibly related to baseline fluctuations. The efficiency of these quality cuts is around $90 \%$.

PMT memory effect. In case of high illumination, the photon rate measured by a PMT contains an intrinsic part which relates to the level of excitation of its glass and photocathode in the past. We refer to such an effect as the PMT memory or historical background. Due to our particular sequence of data taking, where exposure to external source is intermittently turned on and off with a shutter, it is crucial to understand this behavior. For this study, we initially kept the shutter closed for a duration of $200 \mathrm{~min}$, after which it was switched open for $30 \mathrm{~min}$. This sequence was repeated several times and the number of triggers is recorded in intervals of $60 \mathrm{~s}$. The result is plotted in Fig. 4-left, where we can see the photon rate slowly increasing as the photocathode is exposed to some external background. Upon closing the shutter, the rate decays to the nominal value of the internal background ${ }^{5}$, on a timescale of an hour. We apply the following model to describe the output rate $r(t)$ of the PMT given an input signal $s(t)$,

$$
r(t)=s(t)+\alpha \int_{-\infty}^{t} s\left(t^{\prime}\right) \exp \left(-\frac{t-t^{\prime}}{\tau}\right) d t^{\prime}
$$

where in addition to the instantaneous rate we folded an exponentially decaying response of the detector to account for the memory effect. $\alpha$ and $\tau$ are characteristic of the PMT. We obtain these parameters by fitting, with the above model, the average event-rate for each time-window as illustrated in Fig. 4-left. For our PMT, we find

$$
\begin{aligned}
\alpha / \mathrm{min}^{-1} & =0.0170 \pm 0.0003, \\
\tau / \min & =54.124 \pm 1.115 .
\end{aligned}
$$

With these values known, it is possible to fully correct for the memory effect (see Fig. 4-right).

\footnotetext{
${ }^{5}$ We have discarded the hypothesis of a light-leak through the optical blade or defect in the shutter mechanism itself.
} 

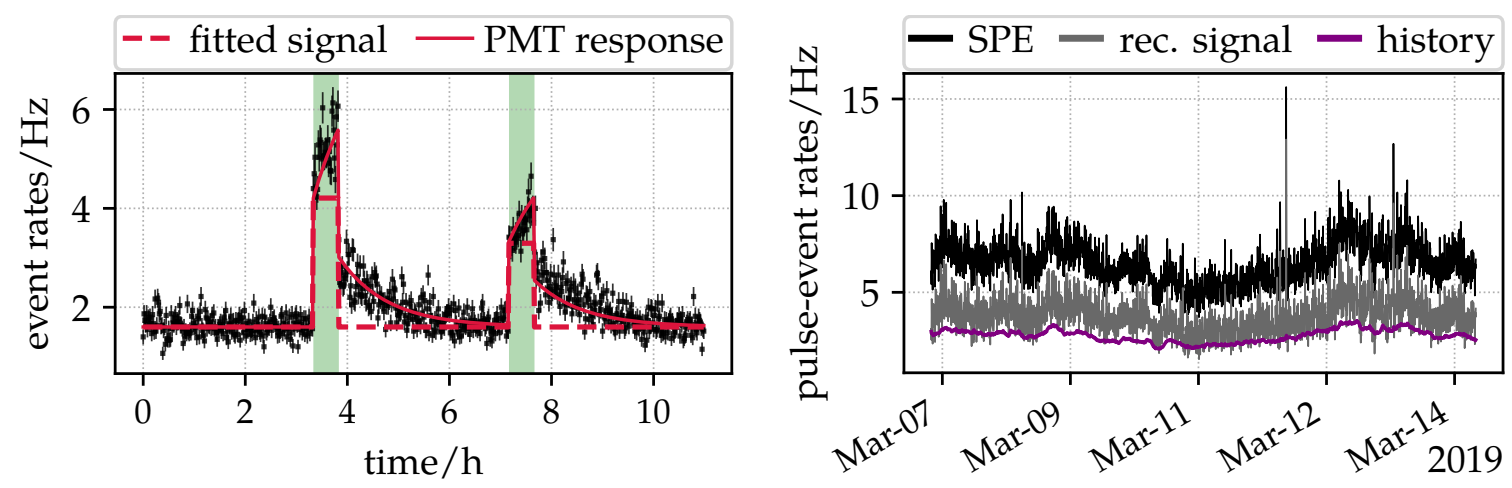

Figure 4: Left: Sample of the trigger rates measured with the sequence closed (200 min) - open (30 min), fitted with the detector response (see text for details). The black markers represent the data and the greenshaded regions correspond to the time interval during which the shutter was open. Right: Example of a signal reconstruction for a one-week data sample taken from the measurement out/open. The label SPE means pulses that pass the quality cuts discussed earlier, and the label history refers the residual rate after disentangling the instantaneous component from Eq. (3.1).

Counting statistics. The event counting exhibits two features which deviates from standard Poisson statistic: (i) a large variance ( 20 times the mean) - (ii) correlated events on short waitingtime scale. The overdispersion can be, for instance, captured by a Gamma heterogeneity in the Poissonian rate. The resulting marginal distribution is a negative binomial whose variance always exceeds the mean. Event arrival-times however depend on the stochastic processes in play. We investigated these processes from an independent run, where the arrival-times of each pulse were also stored. The recored data contain about $4.95 \times 10^{6}$ raw-pulses which are then selected according our true-SPE criteria. In Fig. 5-left we plot the probability integral transform of the durations between successive pulse-events under the assumption of a Poisson process. If the waiting-time were exponentially distributed, this mapping would give a uniform distribution between 0 and 1 . The result however indicates that there is a substantial clustering of correlated events, thereby confirming that a non-Poissonian process is taking place.

Systematics. FUNK operates in very stable environmental conditions ensured by continuous monitoring before, during, and after a complete run. Systematic uncertainties arising from temperature and pressure fluctuations occurring between changes of measurement configuration are estimated to be less than $0.3 \%$ of the difference in - out over the whole run. Likewise, the DAQ implementation offers an excellent timing accuracy with an average up-time of $\Delta t / \mathrm{s}=(60.00138 \pm$ $0.00237)$ per modality per cycle. A systematic shift on the order of this timing uncertainty for each sequence of measurement would result in a systematic bias of $0.004 \%$ on the average count-rate, which remains negligible compared to the counting uncertainty of a month-worth of data-taking. The largest source of systematic uncertainty comes from the mirror reflections. When the PMT is positioned at the center of the mirror sphere with the shutter open, some photons (e.g. from muon hits) may be emitted towards the mirror and get reflected back to the PMT. This effect has been evaluated in run v35 and the result is shown in Fig. 5-right. We measured the trigger-time difference between two pulses and analyzed the time scale which matches the duration that a single photon needs to cover the corresponding distance $(\sim 22 \mathrm{~ns})$. The two observed significant peaks 

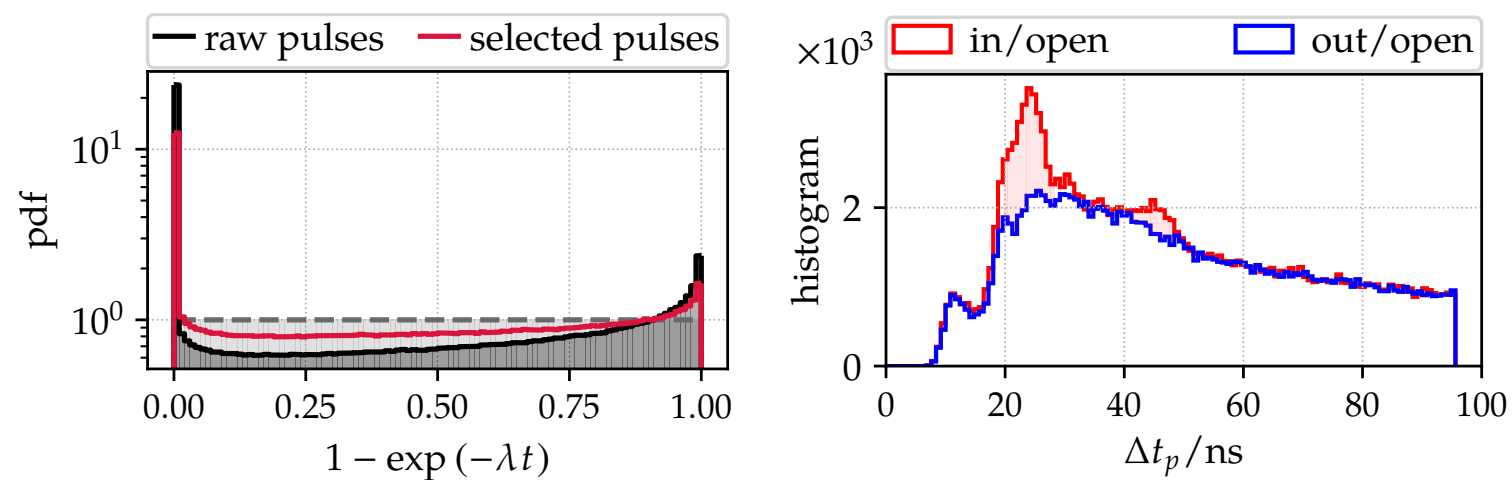

Figure 5: Left: Distribution of the event interarrival times transformed under the exponential cumulative distribution function. The dashed grey line corresponds to the expectation for a Poissonian process. Right: Histogram of the trigger-time difference, $\Delta t_{p}$, between two successive SPE pulses captured within the same trace. The shaded regions correspond to the event excess due to reflections (see text for details).

confirm our hypothesis of photons being reflected once and twice, respectively. The overall contribution of this effect can be estimated with a Monte Carlo simulation, which gives a correction factor of $\sim 0.4 \%$ for the measurement in/open. This results in a systematic error which is of the same order of magnitude as our statistical uncertainty. However, in our conservative analysis, we do not correct for this effect.

\section{Results}

Assuming that the whole CDM in the galactic halo is made of HP condensates, we can express our sensitivity to the kinetic-mixing parameter from Eq. (1.2) such that

$$
\chi=4.1 \times 10^{-12}\left(\frac{\phi_{\text {det }} / q_{\text {eff }}}{\mathrm{Hz}} \frac{m_{\tilde{\gamma}}}{\mathrm{eV}}\right)^{1 / 2}\left(\frac{\eta A_{\text {mirror }}}{\mathrm{m}^{2}}\right)^{-1 / 2}\left(\frac{\left\langle\cos ^{2} \theta\right\rangle}{2 / 3}\right)^{-1 / 2}\left(\frac{\rho_{\mathrm{CDM}}}{0.3 \mathrm{GeV} / \mathrm{cm}^{3}}\right)^{-1 / 2}
$$

where $\phi_{\text {det }}$ is the minimum detectable DM-induced photon rate and $q_{\text {eff }}$ the quantum efficiency of the detector. The parameter $\eta$ encodes the wavelength-dependent reflectivity $R$ of the mirror. For a good reflector, we have $\eta \approx(1+R) / 2$. The angle $\theta$ corresponds to that between the HP field and the mirror surface. If there is no preferred direction, the average orientation is $\left\langle\cos ^{2} \theta\right\rangle=2 / 3$. Finally, we use the value $\rho_{\mathrm{CDM}}=0.3 \mathrm{GeV} / \mathrm{cm}^{3}$ for the local dark-matter energy density.

The potential HP signal is resolved from the difference of single-photon counts inside and outside the region of interest. After data selection and reconstruction, we find

$$
\left(r_{\text {in/open }}-r_{\text {out/open }}\right) / \mathrm{Hz}=-0.0161 \pm 0.0119 .
$$

Nonetheless, it is worth to mention that this negative signal is compatible with the background

$$
\left(r_{\text {in/closed }}-r_{\text {out } / \text { losed }}\right) / \mathrm{Hz}=-0.0278 \pm 0.0112 .
$$

Using Eq. (4.2), we obtain an upper-bound on the magnitude of the mixing parameter such that $\chi \lesssim 6.87 \times 10^{-13}$ at $95 \% \mathrm{CL}$ for the range of masses $1.94 \leqslant m_{\tilde{\gamma}} / \mathrm{eV} \leqslant 8.40$. The final result is shown in Fig. 6. 


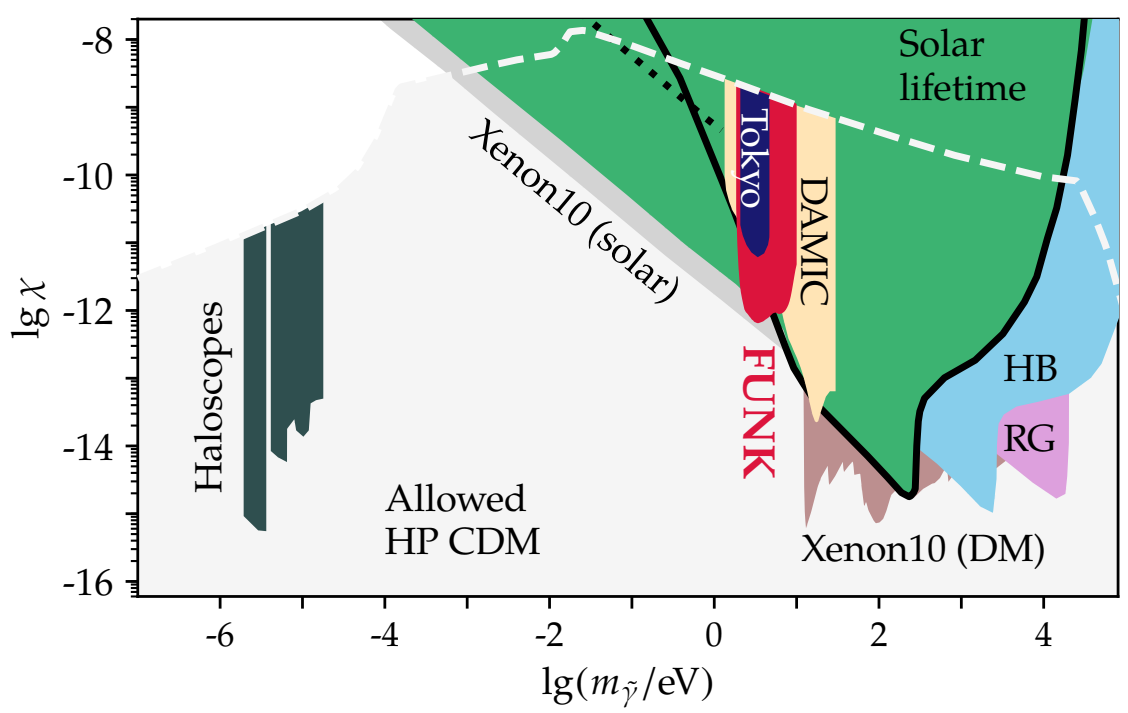

Figure 6: FUNK exclusion limit at 95\% C.L. (red shaded region). The Tokyo limit was obtained by a similar setup but with a smaller mirror [6]. HP bounds from other direct-detection experiments: DM haloscopes [3], DAMIC [10], and Xenon10 (both as DM and as dark radiation emitted by the sun) [11] are also shown in comparison. The regions labeled Solar lifetime, HB (horizontal-branch stars) and RB (red giants) are indirect constraints derived from astrophysical considerations [12]. Finally, the grey-shaded region corresponds to the allowed parameter-space for HP to be CDM [2, 3].

Summary. HP can constitute the entire CDM that our Universe needs. Our experiment searched for HP-CDM with masses $1.94 \leqslant m_{\tilde{\gamma}} / \mathrm{eV} \leqslant 8.40$ but no signal was found. We thus constrained the magnitude of the mixing parameter to $\chi \lesssim 6.87 \times 10^{-13}$ at $95 \% \mathrm{CL}$ within this region.

Acknowledgments. We gratefully acknowledge support from the Helmholtz Alliance for Astroparticle Physics (HAP), funded by the Initiative and Networking Fund of the Helmholtz Association.

\section{References}

[1] J. Jaeckel and A. Ringwald, Ann. Rev. Nucl. Part. Sci. 60 (2010) 405, [arXiv: 1002.0329].

[2] A.E. Nelson and J. Scholtz, Phys. Rev. D 84 (2011) 103501, [arXiv: 1105.2812].

[3] P. Arias, D. Cadamuro, M. Goodsell, J. Jaeckel, J. Redondo, and A. Ringwald, JCAP 06 (2012) 013, [arXiv: 1201.5902].

[4] D. Horns, J. Jaeckel, A. Lindner, A. Lobanov, J. Redondo, and A. Ringwald, JCAP 04 (2013) 016, [arXiv: 1212.2970].

[5] J. Jaeckel and J. Redondo, JCAP 11 (2013) 016.

[6] J. Suzuki, T. Horie, Y. Inoue, and M. Minowa, JCAP 09 (2015) 042, [arXiv: 1504.00118].

[7] D. Veberič et al., [arXiv: 1509.02386].

[8] B. Döbrich et al., [arXiv: 1510.05869].

[9] D. Veberič, A. Andrianavalomahefa, K. Daumiller, B. Döbrich, R. Engel, J. Jaeckel, M. Kowalski, A. Lindner, H.-J. Mathes, J. Redondo, M. Roth, T. Schwetz-Mangold, C.M. Schäfer, R. Ulrich , [arXiv: 1711.02958].

[10] A. Aguilar-Arevalo et al. [DAMIC Collaboration], Phys. Rev. Lett. 118 (2017) 141803, [arXiv: 1611.03066].

[11] I. M. Bloch, R. Essig, K. Tobioka, T. Volansky and T. T. Yu, JHEP 1706 (2017) 087, [arXiv: 1608.02123].

[12] J. Redondo and G. Raffelt, JCAP 1308 (2013) 034, [arXiv: 1305.2920]. 